\title{
Brain Cavernomas Induced By Radiotherapy in the Patient With Acute Lymphocytic Leukemia (ALL)
}

\author{
Babak Alijani, ${ }^{1}$ Hamid Saeidi Saedi,,,* Mohammadreza Emam Hadi, ${ }^{3}$ Shahrokh \\ Yousefzadeh Chabock, ${ }^{3}$ Hamid Behzadnia, ${ }^{3}$ and Adel Kiumarcy ${ }^{3}$ \\ 1 Department of Neurosurgery, Poursina Hospital, Rasht, IR Iran \\ Department of Radiotherapy, Razi Hospital, Rasht, IR Iran \\ ${ }^{3}$ Department of Neurosurgery, Guilan University of Medical Sciences, Rasht, IR Iran \\ *Corresponding author: Hamid Saeidi Saedi, Department of Radiotherapy, Razi Hospital, Rasht, IR Iran. Tel: +98-1333541001, Fax: +98-1333559787, \\ E-mail: hamidsaedisaedi53@yahoo.com \\ Received: April 4, 2015; Accepted: May 15, 2015
}

\begin{abstract}
Introduction: Radiotherapy is considered the main part of the curative and preventive regimes in the pediatric cancer especially has been highly used in acute lymphocytic leukemia (ALL) as prophylaxis. A large number of the radiotherapy secondary delayed effects have been well known.

Case Presentation: In present study, we has introduced a 15-year-old boy with ALL underwent chemotherapy and radiotherapy. Who had generalized tonic-colonic seizure which has been lasted 10 minutes (without any previous history). The patient with ALL ten years ago has been treated by chemotherapy and whole brain prophylactic radiotherapy with dose $1200 \mathrm{cGy} / 120 \mathrm{cGy} / 10$. In his MRI a heterogeneous mass with the dimensions $3 \times 2 \mathrm{~cm}$ in the left frontal lobe with the peripheral edema and after complete the diagnose process the final report of this mass was Cavernoma.

Conclusions: There has been identified a clear link between radiotherapy and brain cavernoma; therefore, it should be considered in differential diagnosis of brain hemorrhagic lesions in each patient with the history of radiotherapy of the central nervous system. This risk is higher when the patient has the history of the brain radiotherapy in childhood.
\end{abstract}

Keywords: Brain Cavernomas; Radiotherapy; Acute Lymphocytic Leukemia (ALL)

\section{Introduction}

Radiotherapy is considered the main part of the curative and preventive regimes in the pediatric cancer especially has been highly used in acute lymphocytic leukemia (ALL) as prophylaxis $(1,2)$. A large number of the radiotherapy secondary delayed effects have been well known. There are clear evidences of Glioma and Miningioma followed by radiation while radiation-induced cavernous angiomas (RICA) has been sporadic and even there has been reported few cases of early appearances of RICA $(2,3)$. In 1994, Ciricillo et al. propounded, for the first time, seven patients with brain cavernoma induced by radiotherapy (4). Since, different cases have been reported in several studies and most of the cases were children with Medulloblastoma (5). In present study, we has introduced a 15-year-old boy with ALL underwent chemotherapy and radiotherapy and it was proved brain cavernoma followed by some signs.

\section{Case Presentation}

The patient, a 15- year- old boy, was referred to referral Radiotherapy center of Razi hospital, Rasht City and complained of the generalized tonic-colonic seizure which has been lasted 10 minutes (without any previous histo- ry). On admission, his GCS was 13 with symmetric movements in four limbs and he has urine incontinence while hospitalizing. The patient with ALL ten years ago has been treated by chemotherapy and whole brain prophylactic radiotherapy with dose $1200 \mathrm{cGy} / 120 \mathrm{cGy} / 10$.

After performing initial treatment and controlling the seizure, brain MRI with and without contrast for the patient was requested. There was observed a heterogeneous mass with the dimensions $3 \times 2 \mathrm{~cm}$ in the left frontal lobe with the peripheral edema (Figure 1). After investigating the spinal cord MRI and CSF, there was not detected any pathological point. The patient with above recognition was treated by craniotomy surgery to drain the mass and it was completely removed. During surgery, the mass appeared to have vascular origin and the case was sent to pathological investigations. The final report of this mass was Cavernoma.

The microscopic description of the lesion in the cases has contained some parts of the cystic structures with fibrotic tissue and adjacent to gliotic brain tissue including the number of macrophages with hemosiderin without any hemorrhage or necrosis and there was not detected some evidence

Copyright (C)2015, Iranian Society of Clinical Oncology. This is an open-access article distributed under the terms of the Creative Commons Attribution-NonCommercial 4.0 International License (http://creativecommons.org/licenses/by-nc/4.0/) which permits copy and redistribute the material just in noncommercial usages, provided the original work is properly cited. 


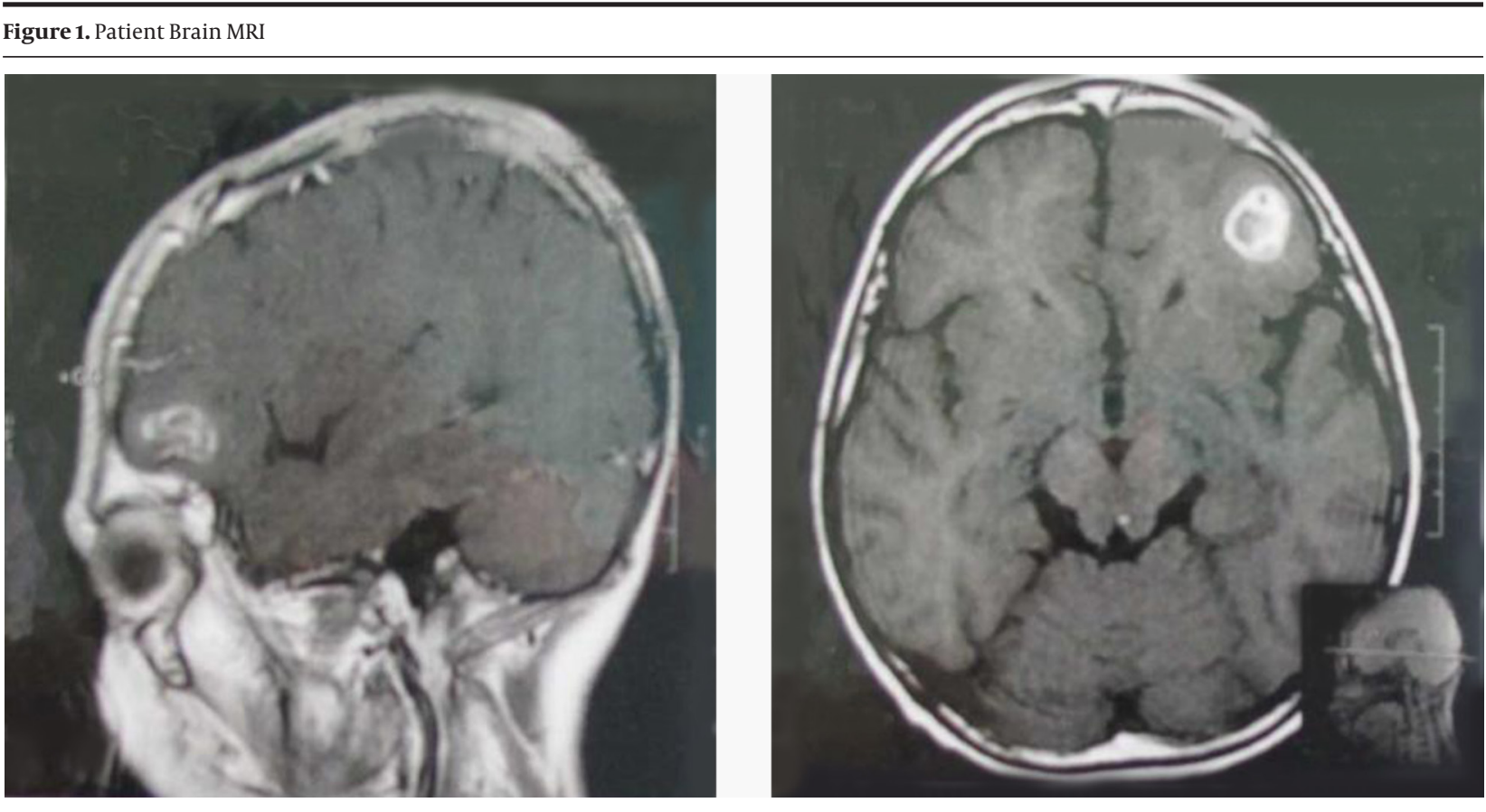

Heterogeneous mass in the left frontal lobe.
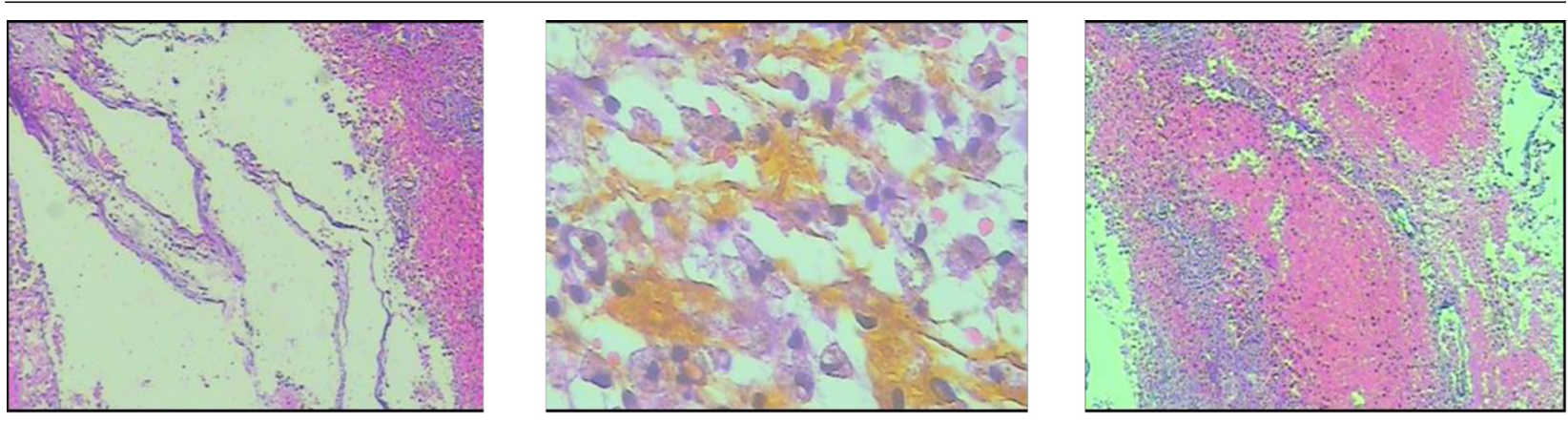

Figure 2. The Appearance of Microscopic Lesion

of malignity. The morphological appearance and the diagnosis of the lesion have been based on cavernoma (Figure 2).

\section{Discussion}

Cavernoma, known Cavernous Hemangioma or Cavernous Malformation, is a vascular lesion in the central nervous system that is composed of dilated capillaries with thin walls without any interstitial brain tissue that has the congenital origin (6). However, there have been observed some sporadic cases and the autosomal dominants with incomplete genetic activities with the frequency $0.5 \%$ (7).

Cavernoma, vascular malformation, is composed of the continuous vascular channels. There may be observed brain parenchyma among channels (8) but, in histological investigations, vessels are hyalinization and secondary changes such as thrombosis is also common. Brain parenchyma adjacent to the lesion is in a gliotic level and it is associated to hemosiderin hemorrhagic extravasation (9). There have been observed some cystic structures with fibrotic tissues and adjacent to the gliotic brain tissue associated to several points of macrophages with hemosiderin in pathological case of the patient's lesion introduced in this paper that are without hemorrhage, necrosis and malignancy evidences which have been cavernoma, based on histological evidences.

According to the reports of Ruggeri et al. (2014), most reported cases of cavernoma induced by radiation have been observed in children with an average age of 11.7 and has been reported in 7 patients over 40 years yet there has not been identified the reason of higher prevalence in children (5). Heckl has assumed that the children with ALL-most prevalence of radiotherapy- are susceptible to cavernoma; although, there is no evidence on this hypothesis in texts. Another assumption discussed by him is that cavernoma has been shaped in the small size be- 
fore radiotherapy but it has been hidden due to radiography and it has grown after radiotherapy and has been observed in MRI (3).

The release of vascular endothelial growth factor (VEGF) due to radiotherapy, has involved in the induction of angiogenesis in which that radiotherapy at first by the fibrosis formation of adventitial layers and endothelial edema causes narrowing the vessel lumen. Reactive angiogenesis due to the increasing release of hypoxy-inducible-factor 1(HIF-1) leads to ischemia and micro infarction which itself cause to VEGF secretion (10). The prevalence of some cavernomas far from the radiotherapy port can be justified to VEGF ability effect further in the distance. VEGF and HIF-1 have been more expressed among very young people. This leads to explain a part of why RICA has been mostly observed in children $(3,10)$.

It is noteworthy that the average of the cavernoma incubation period is $10.3 \pm 1.9$ (5). In the patient introduced in this paper, there has been an interval of 10 years between the radiotherapy and the formation of RICA. In the study of Van Calenbergh, the patient was a young person with neurofibromatosis that has been treated 16 years ago by radiotherapy because of gliomas mesencephali and there has been not found any evidence of cavernoma in early MRI (11).

There has been identified a clear link between radiotherapy and brain cavernoma; therefore, it should be considered in differential diagnosis of brain hemorrhagic lesions in each patient with the history of radiotherapy of the central nervous system. This risk is higher when the patient has the history of the brain radiotherapy in childhood.

\section{References}

1. Baheti AD, Mahore AS, Zade BP, Jalali R. Meningioma and cavernous angioma following childhood radiotherapy.J Cancer Res Ther. 2010;6(3):333-5.

2. Martinez Leon MI. [Early magnetic resonance imaging detection of a cavernous angioma after cranial radiotherapy for an anaplastic ependymoma in a boy]. Radiologia. 2013;55(6):541-5.

3. Heckl S, Aschoff A, Kunze S. Radiation-induced cavernous hemangiomas of the brain: a late effect predominantly in children. Cancer. 2002;94(12):3285-91.

4. Ciricillo SF, Cogen PH, Edwards MS. Pediatric cryptic vascular malformations: presentation, diagnosis and treatment. Pediatr Neurosurg. 1994;20(2):137-47.

5. Ruggeri AG, Donnarumma P, Pichierri A, Delfini R. Two cystic cavernous angiomas after radiotherapy for atypical meningioma in adult woman : case report and literature review. J Korean Neurosurg Soc. 2014;55(1):40-2.

6. Rigamonti D, Hadley MN, Drayer BP, Johnson PC, HoenigRigamonti K, Knight JT, et al. Cerebral cavernous malformations. Incidence and familial occurrence. $N$ Engl J Med. 1988;319(6):343-7.

7. Jain R, Robertson PL, Gandhi D, Gujar SK, Muraszko KM, Gebarski S. Radiation-induced cavernomas of the brain. AJNR Am J Neuroradiol. 2005;26(5):1158-62.

8. Tomlinson FH, Houser OW, Scheithauer BW, Sundt TJ, Okazaki H, Parisi JE. Angiographically occult vascular malformations: a correlative study of features on magnetic resonance imaging and histological examination. Neurosurgery. 1994;34(5):792-9.

9. Gunel M, Awad IA, Finberg K, Steinberg GK, Craig HD, Cepeda O, et al. Genetic heterogeneity of inherited cerebral cavernous malformation. Neurosurgery. 1996;38(6):1265-71.

10. Rivard A, Berthou-Soulie L, Principe N, Kearney M, Curry C, Branellec D, et al. Age-dependent defect in vascular endothelial growth factor expression is associated with reduced hypoxiainducible factor 1 activity. J Biol Chem. 2000;275(38):29643-7.

11. Van Calenbergh F, Demaerel P, Sciot R, van Loon J. Acquired cerebellar cavernous angioma following childhood radiotherapy in a patient with neurofibromatosis type 1. Acta Neurol Belg. 2003;103(2):103-6. 
